\title{
EDITORIAL
}

\section{Celebramos el 30 aniversario de la revista.}

En sí mismo, la conmemoración de su 30 aniversario, no hace a la Revista ni mejor ni peor en comparación con otros cumpleaños inmediatamente anteriores y posteriores, pero resulta una ocasión privilegiada para recapitular sobre lo conseguido, celebrar su salud editorial y posicionarnos frente al futuro. Aunque tendremos que esperar todavía para un estudio histórico, sociológico y bibliográfico en profundidad sobre el papel desempeñado por la Revista dentro de la AEN y su impacto académico y socio-sanitario más allá de la propia Asociación, pero algo había que hacer para resaltar la efeméride. Por ello, solicitamos a cada uno de los anteriores Directores de Publicaciones que seleccionaran un editorial que, de alguna manera y en su opinión, fuera significativo dentro de su mandato al frente de la Revista. Suponíamos que una selección tal nos daría una pequeña idea sobre la diversidad de temas, tanto académicos y asistenciales como sociopolíticos, que la Revista ha abordado en estas décadas, a la vez que serviría como un pequeño homenaje al esfuerzo y entusiasmo mantenido tanto por los sucesivos Comités Editoriales como por los diversos autores. Un trabajo que nos permite ahora disfrutar de una publicación consolidada editorialmente y prestigiada en los sectores asistenciales y académicos más progresistas.

Iniciamos esta pequeña antología de editoriales con un texto obligado, el que firmaba Manuel Desviat inaugurando el número 0 en 1981. Desviat ya opinó sobre lo que supuso ese primer número con ocasión de otra reciente conmemoración, el número 100 (en justicia, el 101) de la Revista. Ahí reflexionaba sobre el papel estratégico que la publicación ocupaba dentro de los proyectos de la AEN para la consecución de sus objetivos reformistas, ya iniciados en 1977 desde el seno mismo de una Asociación que cambiaba sus estatutos para abrirse a la interdisciplinaridad profesional y la descentralización funcional a través de las Asociaciones Autonómicas. Inmersa en el mismo proceso de transición que enfrentaba la sociedad española, la AEN debía "hacerse un hueco en el imaginario social y construir las bases técnicas del proceso asistencial de reforma, era el desafío del momento; para lo que también había que culminar un debate interno, pasar de la denuncia a crear las bases técnicas para la construcción de una nueva realidad psiquiátrica".

A partir de ahí y bajo su dirección editorial desde Madrid, la revista inició su andadura con carácter cuatrimestral, aumentando a partir de 1985 (n 12) su frecuencia de publicación a trimestral. De esa primera etapa Manuel Desviat selecciona el editorial publicado en 1983 con ocasión del XVI Congreso de la Asociación celebrado en Oviedo, presuponiendo en él cierta actualidad para nuestros 
EDITORIAL

días al encuadrar a la Asociación como instrumento científico y de política sanitaria, independiente de la administración. Una Asociación donde producir ideas y herramientas que, apoyadas en una sanidad pública de calidad, posibiliten el desarrollo de la salud mental en sus diferentes niveles.

En octubre $1986\left(n^{\circ} 19\right)$ tomó el relevo al frente de la Revista Teresa Suárez, desde Asturias. En uno de los primeros editoriales de esta época, titulado "Tomemos la palabra", se llama la atención sobre las diferentes secciones que se iban consolidado como expresión de los intereses de los asociados, animando a la participación: La palabra escrita, que deja constancia, aún despierta miedo. El número, mágico, objetivo, indiscutible, científico, es más tranquilizador. No tenemos nada en contra, lejos de ello, salvo la necesidad de traducirlo en palabras que muestren su rigor o descubran que detrás suyo aún siguen escondiéndose viejos mitos marginantes acerca de la enfermedad mental. La selección del editorial realizada en este caso por Teresa Suárez es precisamente el último publicado bajo su dirección: "A modo de despedida", en el que Víctor Aparicio da cuenta de los proyectos y logros de la AEN en ese periodo.

La tercera etapa editorial corresponde a Tiburcio Angosto desde Galicia. Iniciada en abril de 1990 ( $n^{\circ} 33$ ), poco después ( $n^{\circ} 36$ ) se intentó la profesionalización editorial recurriendo con este fin a la Editorial Arán S.A. Se pasó del formato tradicional de $17 \times 24 \mathrm{~cm}$ a los $21 \times 28 \mathrm{~cm}$ con una estética más parecida a las revistas científicas estándar, junto a la inclusión de dos suplementos independientes (psicofarmacología y formación). Además, se incluyó por primera vez publicidad farmacéutica, un aspecto sometido a debate continuo en la historia de la revista. Un ingreso extra dirigido a paliar parte de los costes, que por otra parte se vieron obligatoriamente incrementados con el recurso a la editora externa. La selección del editorial "Siete años de presencia de la A.E.N. en la Comisión Nacional de Psiquiatría" nos recuerda algunos de los momentos más complicados vividos en las relación con la Sociedad Española de Psiquiatría, pero sobre todo las difíciles circunstancias por las que tuvo que atravesar la formación M.I.R. en psiquiatría durante muchos años, en los que además de en los hospitales de la Seguridad Social (o administraciones equivalentes) con capacidad docente, muchos profesionales eran formados en hospitales monográficos concertados o dependientes de las Diputaciones.

En enero de 1994 la revista recuperaba su tamaño original de $17 \times 24 \mathrm{~cm}$. con su único número doble hasta el momento $\left(\mathrm{n}^{\circ} 47-48\right)$, si bien con una estética diferente a la primitiva y que, con muy ligeras modificaciones, es la que se conserva en la actualidad. Igualmente se eliminó la publicidad interior y se rescindió el contrato con la editorial Aran. Al frente de este cuarto periodo editorial, hasta septiembre de 2000, estuvo Fernando Colina. Desde Valladolid, junto a un entusiasta grupo de redactores, algunos de ellos conocidos más tarde como los "Alie- 
EDITORIAL

nistas del Pisuerga", aportaron sus intereses y conocimientos en temas históricos y culturales a las páginas de la revista. En 1995, con ocasión de los 15 años de la revista y una tirada especial de 5.000 ejemplares (habitualmente las tiradas han oscilado de 2.000 a 2.400 ejemplares), editaron un suplemento dedicado a la "Memoria" ( $n^{\circ} 56$ ), que incluía diversos índices de lo publicado hasta esa fecha. El último número de este periodo es un monográfico dedicado a la Historia de la Asociación ( $\left.n^{\circ} 75\right)$. Colina ha elegido un editorial ( $\left.n^{\circ} 49\right)$ firmado por Alberto Lasa en 1994 que, titulado "La psiquiatría de niños y adolescentes, cuestión pendiente", apuntaba a una problemática que todavía tendría que esperar muchos años para empezar a solucionarse.

La quinta etapa, de octubre de 2000 ( $\left.n^{\circ} 76\right)$ a junio de 2006 ( $\left.n^{\circ} 97\right)$, es dirigida por Antonio Díez desde Córdoba. Con ligeras modificaciones en la estructuración de sus contenidos, se suprimieron las secciones Márgenes de la Psiquiatría y Psiquiatría y Cultura, para potenciar la sección Debates donde tendrían cabida entonces los trabajos de reflexión teórica, epistemología y humanidades. En su primer editorial Antonio Díez reflexiona sobre los diferentes contenidos de la revista: a) técnico-profesionales, prioritariamente en el ámbito público y b) de investigación sobre los ámbitos del saber de la salud mental. Aún reconociendo lo borroso de los límites epistemológicos y metodológicos de las disciplinas que aportan su conocimiento a la práctica profesional, opina que las disciplinas de la salud mental exigen una epistemología de niveles, con una metodología diferente según el nivel en cuestión: mientras que las ciencias de la conducta estudian el sentido de ésta y recurren a la comprensión y la interpretación, las neurociencias corresponden a las ciencias naturales, estudian las bases somáticas de la conducta y persiguen explicaciones causales con métodos empíricos. Una dualidad no antinómica siempre tenida en cuenta desde las páginas de la Revista.

De julio de 2006 ( $\left.n^{\circ} 98\right)$ a junio de 2009 ( $n^{\circ}$ 103) retoma la dirección Fernando Colina por segunda vez, lo que, junto a varios de los redactores del Comité Editorial, les convierte en el equipo que más tiempo ha estado no solo al frente de la revista, sino de las publicaciones de la $\mathrm{AEN}$, dejando una impronta difícilmente superable, como por ejemplo la exquisita colección de libros de autores clásicos. La edición de la revista requiere de una dedicación considerable, lo que junto a cierta carestía de materiales y al deseo de economizar gastos aconseja que la tirada se realice de forma semestral, denominando cada número como fascículo y eliminando algunos apartados como las Páginas de la Asociación. Se incluye de nuevo publicidad dirigida a paliar gastos de producción, práctica que debe ser desechada ante las dificultades existentes para asegurar el apoyo económico regular y alejado de personalismos individuales por parte de las firmas farmacéuticas. Durante ese periodo la revista publica su número 100, siendo el del número siguiente el que selecciona Colina en esta ocasión como más significativo. En él, 
EDITORIAL

Alberto Fernández Liria reivindica la "nueva" actualidad de la salud mental, su papel en el debate social, una vez superado el reduccionismo biologicista conservador disparado en los años ochenta, tal y como sucedió en diversos periodos históricos anteriores. A todo ello ha contribuido no solo la falta de resultados en la medida que se llegaron a pronosticar, sino un mayor y mejor conocimiento de las prácticas y recursos asistenciales a nivel global, proponiéndose nuevas formas de actuación y políticas sanitarias dirigidas a la atención en salud mental junto a un mayor reconocimiento a ese derecho.

La séptima etapa, en la que tomamos el relevo desde Bilbao el actual Comité Editorial, empezó en julio del pasado año ( ${ }^{\circ}$ 104). El cambio más ostensible ha sido recuperar la frecuencia trimestral y las Páginas de la Asociación, apostando por las posibilidades que nos brinda Internet, por ejemplo registrando un dominio propio (www.revistaAEN.es) o dando los primeros pasos para la utilización de plataformas editoriales como el OJS. De igual forma que buscaríamos seguir mejorando en lo posible la calidad de la revista, prosiguiendo su adaptación a los requisitos más exigentes de diversos índices o incorporando lectores anónimos para la valoración de los trabajos remitidos para su publicación. Pero en eso andamos todavía, a la vez que auguramos muchos más cumpleaños por delante a la Revista para poder celebrarlo.

Oscar Martínez Azumendi

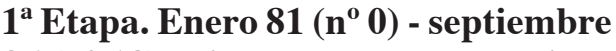
86 ( $\left.{ }^{\circ} 18\right)$. Director Manuel Desviat.

Desviat, M. Editorial $n^{\circ}$ 0. Rev AEN. Septiembreldiciembre 1983. Año I (0): 2-3.

No hay un Saber. Hay saberes parciales, andamiajes conceptuales que nos permiten aproximarnos al conocimiento de las cosas. Más aún en Psiquiatría, en Psicología, en Psicoanálisis, en cuanto que lo psíquico nos introduce en categorías situadas fuera del horizonte de la conciencia, en otra lógica difícilmente asimilable. Este es nuestro primer presupuesto. De ahí partimos, ajenos a todo reduccionis- mo, sea cual sea su signo, su ideología. Una multiplicidad de determinaciones dan cuenta del hecho psíquico, del sufrimiento psicológico y del entramado social que constituye en cada época las Instituciones de la Salud Mental. No pretendemos acotar una, ni convertirnos en portavoces de tendencia alguna. La Revista de la Asociación Española de Neuropsiquiatría quiere ser un espacio abierto a todas las orientaciones y tendencias presentes en la Salud Mental que aporten algo nuevo a las ciencias de la conducta o a la mejora de la situación asistencial, al cambio de las estructuras públicas de atención, a su legislación y administración. No es un eclecticismo. Creemos que todo viaje 
EDITORIAL

teórico, toda aventura intelectual es necesario -no importa que parta de la biología, de la cibernética o del psicoanálisis-, siempre que no sea una simple teorización defensiva. En nuestro país, la teoría y la práctica psiquiátrica permanecen ancladas en un pasado inmovilista, manicomial asistencialmente, provinciano y ramplón en su saber. Es un residuo en nuestra formación social. Una malhadada historia lo explica, lo justifica. Desde la República, donde se oficializa y consolida la labor investigadora y la proyección asistencial de hombres como LAFORA, SACRISTÁN, VILLA VERDE, etc., se ha vivido de prestado, en un escolasticismo irreflexivo, sin preguntas. Unas cuantas fórmulas, tomadas literalmente, escolarmente ritualizadas y repetidas -por lo general cuando ya estaban siendo cuestionadas en su lugar de origen-, han pretendido llenar nuestro vacío teórico. Mera palabrería como producción teórica, arropada a veces con la agresiva y defensiva insolencia con la que se cubre lo deficitario, y una práctica clínica condicionada por una estructura asistencial imposibilitadora de toda actividad terapéutica, salvando raras y particulares excepciones, definen los cuarenta años de reciente pasado. Cualquier cambio, por mínimo y reformista que éste sea, va a poner a la orden del día esta situación plagada de eslóganes, esta insuficiencia. De ahí la necesidad de un espacio, de unas páginas abiertas al debate, a la producción teórica, a la reflexión sobre la práctica, a la información y al análisis de cuanto sucede (y de lo que, premedi- tadamente, no sucede), independientes del juego de poder de las Instituciones: Administración, Partidos, Sindicatos, Escuelas, Iglesias, Gremios, con autonomía aun de la misma Sociedad que lo constituye. Asociación que, a partir de la reciente aprobación de los nuevos Estatutos, se descentraliza en sociedades federadas, en secciones, en comisiones, y da entrada a todos los profesionales de la salud mental, consciente de que ésta no es patrimonio exclusivo de los médicos-psiquiatras. Asociación que se confunde con la misma historia de la Psiquiatría en nuestro país y que, desde hace años, intenta ser el marco científico donde tengan cabida los problemas reales del acontecer psiquiátrico, psicológico, psicoanalítico.

Para terminar, una creencia: la necesidad de la transformación, del cambio radical del orden psiquiátrico vigente, tanto a nivel asistencial y legislativo como en la forma de transmitir y reproducir el saber. Y la convicción de la dificultad de tal empeño en una formación social donde el desarrollo de la salud se enfrenta a poderosos intereses económicos, políticos y profesionales.

Las páginas de esta Revista intentarán dar cuenta de ese acontecer.

Desviat, M. La Asociación en su XVI Congreso. Rev AEN. Septiembre/diciembre 1983. Año III (8): 2-3.

\section{La Asociación en su XVI Congreso}

La A. E. N. viene intentando, desde el Congreso de Sevilla (1977), hito de una nueva etapa, introducirse en el teji- 
EDITORIAL

do social y recuperar las señas de identidad perdidas tras la guerra civil. Origen que nos remite a una asociación científica -aquí el término toma un sentido que trasciende lo meramente académico-, implicada por convicción en la transformación de las estructuras asistenciales y legislativas psiquiátricas yen la producción teórica sustentadora de tal empeño. En estos años de transición democrática, las formas de actuación político-sanitarias se han complejizado, obligando a las instituciones a redefinir sus espacios y sus modos de intervención. La salud ya no es solamente una reivindicación popular con un claro techo político. La salud de un Estado democrático responde a un modelo sanitario, a la elección de un proyecto técnico, económico y social, imbricado en el ámbito de los servicios básicos, con amplia repercusión en toda la formación social y del cual han de dar cuenta, así como de sus formas de implantación, los gobernantes. Este contexto dibuja la función de una asociación profesional como la A. E. N.: instrumento científico y de política sanitaria que ha de constituirse en espacio -con nítida independencia de las Administraciones donde sea posible la producción de ideas, de herramientas que den contenido a las actividades profesionales y posibiliten el desarrollo de la salud mental en sus diferentes niveles, de la gestión a la clínica, de la prevención a la rehabilitación. Pues en el proceso de transformación de la sanidad que la sociedad impone, importa la claridad y capacidad de decisión política, el marco de referencia, el modelo adoptado, mas será el contenido, la maestría, la aptitud de los servicios para dar una cobertura eficaz a la población y su adecuación (prevista) a la relación coste/ eficacia, quienes validen la empresa. Es conocido: las dificultades de la Reforma Sanitaria del Estado de las Autonomías no están sólo en los titubeos de la Administración; sabemos de las resistencias gremiales y empresariales y, sobre todo, de la tremenda ruptura que supone el cambio de orientación exigido por la crisis del modelo médico tradicional -la medicina del órgano, del accidente, y su correlato privilegiado, el hospitalcentrismo -, si el propósito es alcanzar una sanidad pública más integral y positiva para todos los ciudadanos. La asunción de nuevos conceptos de enfermedad y de atención por instituciones y profesionales es, a nuestro juicio, condición de posibilidad de toda reforma que vaya más allá de la mera apariencia.

Así, la A. E. N. mantiene, desde 1977, un desafío político -subversión de la caduca Institución Psiquiátrica, proyecto de integración de la salud mental a la salud general en el marco de unos servicios integrados e integrales de salud pública -y técnico -potenciación de las teorías socio-sanitarias e intrapsíquicas, de planificación y de asistencia al sujeto en conflicto. Cometido que la sitúa -en este sentido hemos trabajado en esta Revista -como espacio para la producción teórica y el debate, la información y la crítica; como entidad facllitadora de materiales de trabajo, útiles para la investigación y la clínica, para la actividad cotidiana y para la lucha por la superación de las categorías y estructuras obturadoras del desarrollo de la salud mental. 
EDITORIAL

En su XVI Congreso, segundo de la nueva etapa, la Asociación se encuentra con una infraestructura y una implantación importantes -quedan lejos las fechas en las cuales Valentín Corcés, como Presidente, y Víctor Aparicio, como Secretario, recogieron, por todo patrimonio, un pequeño libro de actas y un cajón de papeles-pero también ante unos años cruciales, pues en ellos, o así lo creemos, se va a decidir el porvenir de la salud mental en España, un futuro para varias décadas.

\section{$2^{\text {a }}$ Etapa. Octubre $1986\left(\begin{array}{ll}n^{0} & 19\end{array}\right)$ - marzo $1990\left(n^{\circ} 32\right)$. Directora Teresa Suárez.}

Aparicio, V. A modo de despedida. Rev AEN. Enero-marzo 1990. Año X (32): 3-5.

Después del Congreso de San Sebastián (1986), realizamos una editorial con el título de “La AEN y su futuro': señalando el proyecto que se iba a realizar hasta el siguiente Congreso de Madrid y finalizamos con un escueto "en 1990 daremos cuenta..."

Al iniciar este período se vio la necesidad de la modificación del marco estatutario de una asociación que se había complejizado por su crecimiento y su desarrollo autonómico. En un primer momento se realizó un pacto autonómico entre la AEN y cinco asociaciones autonómicas que permitió regular las relaciones, el reparto económico y la doble adscripción de los socios. Este pacto sirvió de base a los estatutos que, por fin, quedaron aprobados en las Jornadas de Santiago de Compostela (1989) y ha permitido la incorporación de dos nuevas asociaciones autonómicas (Balear y Castellano-Leonesa). Paralelamente hemos vivido la situación conflictiva de ver cuestionada nuestra representación en la Comisión Nacional de Especialidad de Psiquiatría. Esta experiencia ha estado íntimamente relacionada con la historia de recelo mutuo entre las dos sociedades nacionales de psiquiatría (AEN y SEP). En el Simposium Mundial de Granada (1989) la AEN inició, con la invitación a la participación de la SEP, el acercamiento entre dos sociedades abocadas al diálogo. Hoy la normalización de las relaciones ha permitido que la AEN participe en el próximo Congreso de la SEP (Salamanca-1990) y que ambas sociedades tengan representación en la Comisión Nacional de Psiquiatría.

En esta Comisión nuestra asociación ha desarrollado una política de extender el sistema MIR al mayor número posible de servicios y de cuidar por la calidad de este sistema. En la otra cara de la moneda ha estado el esfuerzo, junto con el Colegio Oficial de Psicólogos, de propiciar el reconocimiento oficial del PIR y de buscar una solución al problema "histórico" de los becarios.

El debate científico y asistencial han sido el eje de las sucesivas Jornadas celebradas en estos tres años, que se han visto complementadas con un conjunto de seis libros que han recogido materiales presentados en las mismas (Jornadas de Infantil en Gijón, Jornadas Nacionales de Canarias y Congreso de Madrid), e incluso la recopilación de una ponencia (Sevilla-1977) de Carlos Castilla del Pino, como un elemento 
EDITORIAL

más del homenaje que le rindió la Asociación y que ha tenido como colofón la presentación de su candidatura al Premio "Príncipe de Asturias".

Este debate también ha encontrado reflejo en la línea editorial de nuestra revista, y en las acciones que se han realizado puntualmente ante situaciones asistenciales o legislativas que atentaban contra derechos de los usuarios, de los profesionales o provocaban frenos a reformar en marcha (Penitenciarios de Fontcalent y de Carabanchel, Hospital Psiquiátrico de Lugo, situación de la red de Barcelona, crisis de Alicante, caso Ibiza...).

Se consiguió dar un salto tanto cualitativo como cuantitativo en la política de relaciones internacionales. Por primera vez, se establecieron relaciones fluidas con la Asociación Mundial de Psiquiatría, permitiendo la celebración del Simposium Mundial de Granada, y la asistencia de una delegación al Congreso Mundial de Atenas donde quedó aprobada una moción presentada por la AEN para la no colaboración de los psiquiatras en procesos a oligofrénicos en los que se estableciera la pena de muerte. Como diversificación de esta política nos integramos en la Federación Mundial de Salud Mental y establecimos relaciones con diversas asociaciones nacionales (Asociación Francesa de Psiquiatría, Asociación Portuguesa de Psiquiatría Social, Asociación Alemana de Psiquiatría Social, Asociación Griega de Desinstitucionalización, Psiquiatría Democrática Italiana, Asociación Americana de Psiquiatría, Asociación Centroamericana de Psiquiatría, Asociación Argentina de Psiquiatría y Asociación Marroquí de Psiquiatría). Con esta última, las relaciones permitieron hacer gestiones para seguir apoyando la libertad del psiquiatra marroquí M. Jaaidi.

Este crecimiento de actividades ha sido paralelo a un crecimiento de socios, hoy casi 1.400 , que ha necesitado de una mejora de la infraestructura y de la gestión (informatización y sistemas de control presupuestario), pero que también ha cristalizado en una asociación más heterogénea. Al final del período pudimos dar expresión a la diversidad de nuestra vida asociativa, por primera vez, a través de un proceso electoral democrático y secreto que había sido reclamado con intensidad en aquella, hoy histórica, Asamblea de San Sebastián.

Al final la homogeneidad asociativa era un espejismo que deja paso a un futuro más complejo, pero que por su diversidad será más enriquecedor.

\section{$3^{\text {a }}$ Etapa. Abril $1990\left(n^{0} 33\right)$ - diciem- bre $1993\left(n^{\circ} 46\right)$. Director Tiburcio Angosto.}

Angosto, T. Siete años de presencia de la AEN en la Comisión Nacional de Psiquiatría. Rev AEN. Abril-junio 1992. Año XII (41): 95-96.

En Junio de 1985 durante las VI Jornadas de la AEN. que se celebran en Vigo, el Dr. V Aparicio recibió el encargo del Dr. J García González, presidente de la Asociación en aquellos momentos, de incorporarse a la Comisión 
EDITORIAL

Nacional de Psiquiatría (C.N.P.) dado que ese mismo día le llegó la noticia de que el Ministerio de Sanidad había corregido el error de nombrar a los dos representantes de la SEP.: Drs. Conde y López Ibor, a favor de la representación de la AEN. El Dr. V Aparicio abandona las Jornadas y vuela hacia Madrid para incorporarse a la Comisión Nacional que se reunía al día siguiente. Permanece como miembro hasta 1986. Cuando sale elegido Presidente de la AEN. lo sustituye quien escribe estas líneas.

Como es bien sabido, la SEP., presenta un contencioso contra el Ministerio por este nombramiento, que tras perder en los tribunales ordinarios, gana, tras sentencia del Supremo, en Octubre de 1990.

En Marzo de 1992, el Ministerio, en cumplimiento de dicha sentencia, nos comunica mi cese como miembro de la Comisión Nacional en representación de la A.EN., para ser sustituido por el Dr. JJ. López-Ibor Aliño. (Ver cartas que se publican en esta revista).

Así pues, nuestra asociación, ha tenido representación desde que se inició la andadura del Real Decreto de Especialidades 127/84. Primer Decreto que proporciona una configuración democrática a las Comisiones Nacionales.

Hacer un balance de estos años no es fácil ya que han estado plenos de renovaciones.

El primer objetivo que tuvo la Comisión fue la elaboración de un Programa para la Formación M.I.R. que se apartara de los academicismos del anterior y estuviera más centrado en las posibilidades de los servicios que iniciaban su andadura en proyectos de reforma de la asistencia psiquiátrica. Dicho programa que supuso un gran avance, actualmente en la Comisión Nacional se está valorando reformar sobre todo en lo relativo al desarrollo de las Areas de Capacitación y a los periodos de formación y rotaciones.

Se elaboraron las "Normas para la acreditación de unidades docentes para la formación M.I.R.”. Con estas normas, que se revisaron a lo largo de este tiempo, varias veces, se consiguió incorporar al sistema de formación muchos hospitales, con excelentes profesionales, que hasta entonces ni siquiera se habían planteado la posibilidad de ser docentes. Así se consiguió pasar de las 59 plazas de 1985 a las 131 acreditadas que hay actualmente y que si bien no cubren totalmente las demandas de profesionales para dejar de ser una especialidad deficitaria, proporcionan un buen alivio para los desarrollos de los procesos de reforma.

En Junio de 1986 tuvo lugar el examen de la transitoria 4.a del RO. 127/84. Fue laborioso y complicado.

Tras examinar a más de 200 aspirantes al título, no dejó contentos a nadie: ni tribunal ni aspirantes. La CN-P (la AEN. así lo defendía también) quería aumentar el número de títulos, dado lo agobiante del déficit de psiquiatras por aquella época pero el Ministerio no quiso salirse del corsé del 5\% que proponía el RO. ni utilizar la argucia de contemplar la posible complementariedad con otras especialidades que no cubrían su cupo. No obstante la C.N.P. aprobó más del $5 \%$ pero no les concedieron el título a todos. 
Algunos de éstos eran becarios que habían tenido una formación igual a la M.I.R., que están llevando a cabo, en la actualidad, tareas asistenciales similares a los psiquiatras, pero que no tienen posibilidad alguna de obtener el título por esta vía y cuyo problema sigue aún candente.

En la última entrevista con el Ministerio de Sanidad, la Junta Directiva actual le volvió a plantear la necesidad de buscar una solución a este colectivo, pareciendo detectarse de nuevo un gran interés por solucionar este problema.

Es necesario también decir que la C.N.P. aceptó que la nominación para los sistemas docentes de los M.I.R. dejen de hacerse obligatoriamente por el nombre de los Centros Hospitalarios Psiquiátricos tal y como proponía en la campaña de recogida de firmas, que la AEN. llevó a cabo en Mayo de 1989. En la última convocatoria ya aparecen algunos servicios en esta forma nominados.

Es preciso decir que en Mayo de 1990 fue elegido el representante de la AEN. como secretario de la C.N.P. al mismo tiempo que el Dr. A. Espino como Presidente.

Sin embargo la mayor parte del trabajo en la C.N.P. tuvo que ver con asuntos de:

-Trámite: como la acreditación de nuevos servicios para la formación M.I.R.., cambios de especialidad, problemas en relación a defectos en la formación por incumplimiento del programa, etc.

-Opinión: como el reconocimiento de la especialización en Psiquiatría Infanta-Juvenil, la creación del Sistema P.I.R, aún pendientes, etc.
-Asesoramiento: como el siempre problemático tema de las homologaciones por estudios en el extranjero. Desde Octubre de 1990, que fue cuando se hicieron masivas las peticiones de homologaciones, hasta Febrero de 1992 se han visto 84 expedientes y recursos en siete reuniones, sin contar las solicitudes' de titulación por el Artículo 18 del RO. 127/84.

Se ha echado en falta algo imprescindible en el seguimiento de la formación de los futuros psiquiatras: auditorías de los centros acreditados.

Sabemos que en muchos centros acreditados no se está siguiendo el programa docente establecido, que muchos no cumplen los mínimos para la acreditación y que otros cambiaron las condiciones que permitieron dicha acreditación y sin embargo la siguen manteniendo.

Es preciso hacer auditorías docentes que nos permitan saber el grado de cumplimiento de la normativa establecida y poder hacer correcciones para conseguir el perfil más óptimo posible de los profesionales que se pretenden.

Curiosamente las denuncias por incumplimiento de programa, de los M.I.R. que han llegado a la C.N.P. han sido pocas, de tal forma que solamente en un caso se ha recomendado al Ministerio la "congelación" de la acreditación docente de un centro.

Resulta prácticamente imposible hacer un análisis objetivo de la posible incidencia que hemos tenido los que hemos representado a la A.EN. en la Comisión Nacional, sobre las decisiones que se han tomado, que por otra parte 
EDITORIAL

han sido siempre mediante consenso. Pero no resulta difícil rastreando en las actas, encontrar, en dichas decisiones, aspectos de lo que podrían ser las señas de identidad de la AEN.:

-Formación más práctica que teórica.

-Facilitar el acceso a la acreditación docente de centros no universitarios.

-Sistema de formación no hospitalocéntrico con rotaciones extrahospitalarias.

-Programa con mínimos fáciles de cumplir, etc.

No podemos decir que estos aspectos hayan sido defendidos solamente por los representantes de la A.E.N. ya que en la C.N.P. han estado otros miembros de nuestra Asociación representando a los Ministerios tanto de Sanidad como de Educación, aunque sí tenemos el ejemplo de la C.N.P. previa al decreto 127/84 cuya labor, que podríamos catalogar de excesivamente academicista, fue muy distinta a la llevada a cabo por las posteriores.

Decíamos antes que las decisiones de la C.N.P. se hacían por consenso. En lo que se refiere al tiempo que yo he permanecido en ella no ha habido graves tensiones que conllevaran ánimo de ruptura. Ha habido disparidad de criterios y creo que siempre se han saldado con una solución pactada, aceptada y defendida por todos. Por ello no se entiende el empeño de la Sociedad Española de Psiquiatría en llevar adelante el recurso contencioso (el cual se nos dijo personalmente que había sido retirado del Supremo) que ha acabado por apartarnos de la C.N.P. sobre todo cuando las relaciones entre ambas Asociaciones se estaban normalizando y creo que a ello contribuyó, en parte, la convivencia dentro de la misma C.N.P.

Han llegado a nuestros oídos varias versiones, pero como siempre un rumor no es verdad hasta que una firma lo autoriza y la AEN. no ha recibido ninguna explicación del cambio de actitud de la SEP

Se ha dicho que el mantenimiento del recurso fue un "lapsus" provocado por el cambio de Junta Directiva de la SEP .. pero ¿cómo se explicaría la posterior carta a los Consejeros de Sanidad de las CCAA. para que impidan la representación de la AEN. en los tribunales para la provisión de plazas vacantes?

Alguien también habló de una posible revancha por nuestra abstención en la elección del Secretario General de la Asociación Mundial de Psiquiatría (WPA).

Otra de las versiones, más mantenida en el tiempo, es la de que la agresividad del recurso no va dirigida contra la AEN. sino contra el Ministerio de Sanidad, lo cual no deja de ser "simpático" cuando los "Fundamentos de Derecho" que la SEP presenta en el Contencioso es que la AEN. "no reúne las condiciones necesarias para tener un vocal en la aludida C.N.P."

Parece claro que hubo intencionalidad de apartarnos de la eN.p y cierta alevosía basada en la incentivación de confianza.

¿Qué significa esta pérdida? Tal vez, no mucho más que un lugar para tratar de modificar los cauces por donde 
se mueve la transmisión del Saber, o tal vez simplemente un referente de asesoramiento tanto para los compañeros que pretenden iniciar la acreditación docente del servicio donde trabajan, como para los colegas hispanos que pretenden homologar su titulación.

Sabemos que nadie se inscribe en la AEN. por que está representada en la C.N.P., pero también sabemos que una asociación que tiene como seña de identidad la preocupación por la calidad de la asistencia no puede estar ajena al proceso por el cual se van a elaborar las directrices de formación de los futuros profesionales que la van a llevar a cabo o simplemente no puede estar ausente de los foros donde se discute el futuro de aquello que es el objeto de su definición.

Como es sabido y conocido: donde la AEN. esté ausente otros van a reclamar ese lugar.

\section{$4^{\text {a }}$ Etapa. Enero 1994 ( $n^{0}$ 47-48) - septiembre $2000\left(n^{0} 75\right)$. Director Fernando Colina.}

Lasa, A. La psiquiatría de niños y adolescentes, cuestión pendiente. Rev AEN. Abril-junio 1994. Año XIV (49: 211-213.

Cuando se inició la reforma psiquiátrica, surgieron esperanzadas expectativas entre quienes trabajaban en el campo de la salud mental con niños y adolescentes. Ahora ha pasado suficiente tiempo como para intentar un balance de la situación actual. Nadie discute los logros -por supuesto me- jorables-conseguidos en el terreno de la asistencia psiquiátrica para adultos, como nadie ignora, igualmente, que el crecimiento no ha sido semejante en la asistencia destinada a niños y adolescentes. Tanto los profesionales de la salud mental que trabajan con ellos, como los que conviven con los mismos en otros lugares (escuelas, servicios sociales y de protección a menores), y los expertos y/o responsables de la planificación de los recursos, todos coinciden a la hora de señalar la carencia de servicios y estructuras de tratamiento y acogida específicos.

Todavía recientemente se afirmaba la conveniencia de no psiquiatrizar a niños y adolescentes y de optar por modelos más integradores. Hoy sabemos que esta opción, junto con innegables progresos en la integración escolar de ciertos niños, también ha descargado sobre otros profesionales e instituciones (sobre todo de las llamadas de educación especial) nuestras propias responsabilidades terapéuticas. Que nadie resucite otra vez, y menos en lo que se refiere a niños y adolescentes, el riesgo de excesiva psiquiatrización. Nadie se ha ocupado más de evitar posibles abusos que los propios profesionales que, además, no están precisamente ávidos por ampliar su ya excesiva clientela. Cabe recordar, sin ánimo de irritar a nadie, que para desarrollar actividades e instituciones psiquiatrizadoras no hace falta ser psiquiatra. Para practicar la peor psiquiatría posible, basta con desentenderse del funcionamiento mental del sujeto. Ya no hace falta ni hablar con él ni escuchar lo que dice. 
EDITORIAL

Cabe preguntarse, igualmente, si no está sucediendo, en el terreno de las psicosis y de otros trastornos psíquicos graves -que, recordémoslo, también afectan a niños y adolescentes-, algo parecido a lo que ya ocurrió con la asistencia al deficiente mental desde que la psiquiatría, condicionada por su lúgubre experiencia previa, se desentendió del problema. Y podemos decirlo sin eufemismo porque hasta la OMS nos sugería, no hace mucho, la necesidad de una reimplicación de la sanidad en una situación problemática que abandonó voluntariamente.

Las carencias asistenciales actuales, sobre todo en cuanto a estructuras intermedias, y nuestra cultura asistencial, caracterizada por el descubrimiento de un nuevo espacio socio-sanitario, están favoreciendo una nueva tendencia: la derivación de lo que siempre fueron responsabilidades terapéuticas hacia instituciones de acogida, integración escolar/social y reinserción. Es evidente la necesidad de éstas como complemento de los servicios sanitarios, pero todo parece apuntar a que la actual crisis del estado de bienestar empuja a sacrificar el desarrollo y el coste de servicios específicos para niños y adolescentes en favor de otras prioridades asistenciales. Se cede aquí un espacio que siempre ha pertenecido a la salud mental a otras instituciones, concertadas o subvencionadas con dinero público, que pueden jugar un papel sustitutivo de opciones asistenciales sacrificadas antes de nacer.

Puede ocurrir que, de golpe, quienes viajaban en el furgón de cola de la reforma, el de la psiquiatría infantil, mientras estaban esperando pacientemente el momento de llegar al andén donde repartían las riquezas que nuestra nueva abundancia prometía, queden destinados a la resignación. La locomotora no da más de sí y el maquinista decide que el último vagón sea desenganchado y pase a vía muerta. Sin embargo, los numerosos profesionales que trabajamos en este campo no llegamos a creer que las realidades económicas obliguen a declarar utópica la necesidad de desarrollar medios asistenciales imprescindibles. Si todo el mundo acepta, hablando de la salud mental, la necesidad de prevención, es difícil entender que ello no se traduzca en la decisión de destinar recursos prioritarios para la misma en el campo de la infancia. A no ser que se piense que la infancia no es un campo prioritario de actividades preventivas o que se opine que éstas no son rentables o bien porque en la noción de prioridad y de rentabilidad se infiltren otras cuestiones.

Por ejemplo: compárese la dimensión de nuestra población de niños y adolescentes que tiene problemas de salud mental con la de los toxicómanos. Compárese, seguidamente, la cantidad de recursos destinados a unos y a otros. ¿Cómo medir su rentabilidad? ¿Por su eficacia preventiva o terapéutica? Sin ánimo de provocar: ¿no será la rentabilidad derivada de mitigar la presión social la que decide cuáles son las opciones asistenciales a priorizar? ¿No obtendría la psiquiatría más recursos para los niños y adolescentes si su sintomatología les llevara a asaltar bienes y viandantes más a menudo? Sabido es, también, que las leyes inmisericor- 
EDITORIAL

des de la inercia administrativa hacen que, en toda dotación presupuestaria, la atracción que hacia sí mismas ejercen las instituciones preexistentes sea directamente proporcional a su influencia gravitatoria -constituida por su peso arquitectónico y por su masa salarial y sindical-o Lo cual dificulta grandemente el desprendimiento de nuevos satélites con influencia atractiva propia. De nuevo la vieja y sabida historia de que innovar es difícil.

Existen, por lo tanto, varias y pesadas razones para que la asistencia psiquiátrica destinada a niños y adolescentes no cuaje en nuestro país con la fuerza con que lo ha hecho en casi todos los países europeos. Su elucidación y discusión merecería una reflexión más amplia que la que este editorial permite. Lo que está muy claro es que aún queda mucho por hacer, en casi todos los lugares de nuestra geografía, para completar una asistencia adecuada. Como bien saben los profesionales que ejercen en este campo, y algunos suman muchas décadas de experiencia, se necesitan servicios ambulatorios específicos articulados con los destinados a adultos, sin que sean éstos los que se vean obligados a improvisar, con más o menos imaginación, cómo suplir las carencias actuales. Servicios que estén vinculados también con la red de atención primaria y con antenas hospitalarias que les articulen con la pediatría. Dotados, además, de las imprescindibles estructuras intermedias que les permitan relacionarse y complementarse, si es posible hasta en su financiación, con lo que oferten los servicios sociales, escolares y otros.
Carecemos todavía de suficientes lugares con programas de formación adecuada, aunque existan algunos, y falta en todos ellos la sanción de una titulación reconocida que, muy probablemente, les va a ser exigida a las autoridades sanitarias de nuestro país como consecuencia de la aplicación de los acuerdos europeos de libre circulación de profesionales, que obligarán a homologar las vías y programas de acceso a toda especialidad. Programas que exigirán como mínimo varios años de formación en centros especializados de niños y adolescentes, tras el paso obligado por el tronco común de la psiquiatría, y con vías de acceso previstas para quienes provengan de la pediatría.

Deberíamos, por último, evitar un autoengaño fácil. Me refiero al consistente en creer que podemos resolver nuestros problemas asistenciales sin crear servicios específicos. Lograríamos, con él, ocultar lo inocultable, que no se ha hecho ni se está haciendo todo lo que nuestro momento histórico, cultural y económico permitía y permite.

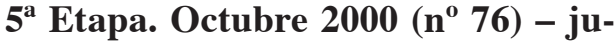 nio $2006\left(n^{\circ}\right.$ 97). Director Antonio Díez Patricio.}

Díez Patricio, A. Editorial. Rev AEN. Octubre-diciembre 2000. Año XX (76): 3-4

La Revista de la AEN, como órgano de expresión de una asociación de profesionales de la salud mental, recoge publicaciones acerca de dos tipos de actividades: a) las meramente profesionales, es decir, técnicas, que en nuestro 
EDITORIAL

caso suelen referirse al ámbito de la asistencia pública, con la que la AEN, estatutariamente, está comprometida; b) las de investigación sobre los ámbitos del saber en los que se basa la salud mental. En lo que a este segundo grupo respecta, las posibilidades son ingentes, dada la heterogeneidad y multiplicidad de las disciplinas que aportan sus conocimientos a nuestra práctica profesional. Estas disciplinas pueden ser divididas en tres grupos:

1) Ciencias biológicas (bioquímica, fisiología, psicofarmacología, etc.) o ciencias de los procesos lógicamente necesarios de la conducta.

2) Psicología y psicopatología o ciencias de la conducta y del sujeto de la conducta.

3) Ciencias sociales (sociología, antropología social) como ciencias de la comunidad en la que tiene lugar la génesis del sujeto y se produce su conducta.

Ahora bien, estos tres grupos constituyen, además, tres niveles de aproximación al estudio de la conducta. Ateniéndonos a la clásica división de las ciencias que estableciera Dilthey, esto es, ciencias de la naturaleza y ciencias del espíritu, y a sabiendas que los límites epistemológicos y metodológicos entre ellas son borrosos y en muchos casos se solapan, el primero de los grupos constituye el estudio de las condiciones necesarias, básicas, aunque no suficientes, para que se produzca la conducta. Se trata de las bases somáticas de la misma y su modelo es el de las ciencias naturales, que tiene en la física su paradigma. Los dos grupos restantes estudian distintos niveles de organización de la conducta, su modelo es el de las ciencias humanas y su paradigma sería la psicología.

Como ciencia, la psicología plantea numerosos problemas epistemológicos. Si una ciencia se define por su objeto, el de la psicología, que es la conducta, aún no está claramente delimitado. Existen tantas psicologías como conceptualizaciones de la conducta se consideren o aspectos de la misma se estudien. Si bien es verdad que las aportaciones procedentes de algunas de ellas (como la psicología dinámica, la cognitiva, etc.) son valiosas, la confusión epistemológica es grande, particularmente en lo que concierne a la psicología como fundamentación teórica de la psicopatología. Como es sabido, la carencia de un modelo que dé cuenta de todas las conductas posibles, normales y anómalas (y, dentro de éstas, las patológicas) ha llevado a algunos, en pro de una mayor fiabilidad del diagnóstico, a plantear una psiquiatría "ateórica", es decir, sin fundamentación psicológica y psicopatológica. En virtud de la mayor comunicabilidad lograda, es evidente que se han facilitado algunas facetas de la investigación, como es la epidemiología clínica, la psicofarmacología, etc., pero, si se toma como la única psiquiatría posible, aparte de la contradicción lógica que implica, el empobrecimiento es enorme. Porque, aunque supuestamente ateórica, esta psiquiatría esconde un reduccionismo biologista, que deja traslucir el intento iniciado en el siglo XIX de encuadrar las alteraciones de la conducta en el modelo médico (anatomo y fisiopatológico), olvidando que el diagnóstico psiquiátrico es psicopatológico y que, por tanto, todo 
progreso en psiquiatría, siendo ésta una aplicación práctica, ha de partir de la indagación psicopatológica.

La aprehensión científica de la conducta exige la consideración de un dualismo epistemológico y metodológico. Las ciencias positivas han de dar cuenta de las bases somáticas de la conducta. Pero la conducta, entendida como actividad con sentido -esto es, motivación, intencionalidad, etc.- supone un sujeto que la realiza, cuyo estudio exige una metodología diferente, de carácter interpretativo. No hay que pensar que este dualismo epistemológico y metodológico implica un dualismo ontológico. Una visión monista del sujeto no tiene por qué conllevar que los métodos de estudio de sus constituyentes hayan de ser, asimismo, monistas. En el estudio del sujeto es preciso diferenciar los hechos somáticos de los psíquicos. Para los primeros, la metodología es cientificopositiva; para los segundos, es comprensiva, es decir, interpretativa (hermenéutica). Las disciplinas de la salud mental exigen una epistemología de niveles, con una metodología diferente según el nivel en cuestión: mientras que las ciencias de la conducta estudian el sentido de ésta y recurren a la comprensión y la interpretación, las neurociencias corresponden a las ciencias naturales, estudian las bases somáticas de la conducta y persiguen explicaciones causales con métodos empíricos.

¿Quiere esto decir que las primeras hayan de escorarse hacia la especulación teórica? Si bien es cierto, y más en lo que a las ciencias humanas se refiere, que nuestra teoría de la realidad determina nuestra aprehensión de ella, en todos los casos la actitud racional - la ciencia es paradigma de la racionalidad - consiste en la coherencia lógica de las teorías y la confrontación de éstas con los hechos de la realidad. La excesiva teorización, no acompañada de contrastación alguna con la realidad, suele conducir a abstrusas lucubraciones carentes de rigor y pragmatismo. Por el contrario, acercarnos a los hechos sin una teoría previa acerca de ellos constituye una estéril práctica sin posibilidad de progreso.

Recientemente hemos celebrado el 75 aniversario de la AEN y se ha publicado el número 75 de esta revista, que cumplirá pronto los 20 años de existencia. Se trata de una revista ya consolidada, vehículo de expresión de una asociación multidisciplinar de profesionales de la salud mental. En esto, ocupa un lugar bien definido entre las publicaciones científicas de nuestro país. En el editorial de su número 0, su entonces director, M. Desviat, escribía las siguientes palabras, que ahora hago mías: "No hay un saber. Hay saberes parciales, andamiajes conceptuales, que nos permiten acercarnos al conocimiento de las cosas". Y más adelante añadía: "Este es nuestro primer presupuesto. De ahí partimos, ajenos a todo reduccionismo, sea cual sea su signo, su ideología". Para continuar: "La Revista de la Asociación Española de Neuropsiquiatría quiere ser un espacio abierto a todas las orientaciones y tendencias presentes en la Salud Mental que aporten algo nuevo a las ciencias de la conducta o a la mejora de la situación asistencial, al cambio de las estructuras públicas de atención, a su legislación y administración." Que así sea. 
EDITORIAL

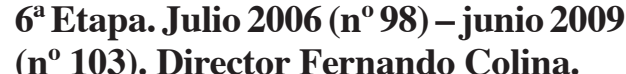

Fernández Liria, A. La nueva actualidad de la salud mental. Rev AEN. Fascículo 1, 2008. Año XXVIII (101): 3-5.

La salud mental y la atención a los trastornos mentales han ocupado un lugar destacado en el debate social en diversos momentos históricos. Así, por ejemplo, la introducción del psicoanálisis supuso una auténtica conmoción en los inicios del siglo veinte, las aportaciones de los culturalistas fueron best sellers en los años cincuenta y la voluntad de descifrar el tipo de cuestionamiento de los usos sociales que encerraba la locura lo fue en los sesenta y setenta de la mano de los llamados antipsiquiatras, de los reformadores de la psiquiatría o de Michel Foucault y seguidores ya en los sesenta y setenta.

En los años ochenta las referencias a la salud o los trastornos mentales fuera de los ámbitos especializados pasaron a ser meramente marginales. A la sombra de las grandes revoluciones conservadoras, la atención a la salud mental dejó de ser considerada un desafío para el Estado del Bienestar o una fuente de inspiración para el pensamiento crítico, para pasar a ser observada únicamente como un potencial mercado en el que la industria podría realizar beneficios.

El pensamiento psiquiátrico y la actividad de los psiquiatras pasaron a supeditarse de un modo absoluto a este fin. La salud mental dejó de ser pensada como un logro difícilmente construido, y pasó a considerarse un estado natu- ral sólo amenazado por alteraciones bioquímicas del funcionamiento cerebral, pues se esperaba que el desarrollo paralelo de las neurociencias -que hay que decir que se ha producido a pesar de los psiquiatras y no gracias a ellospudiera explicar las enfermedades. Los psiquiatras pasamos a ser prescriptores $\mathrm{y}$, en todo caso, testigos y voceros de las bondades de los remedios que se disputaban el nuevo mercado. Nuestro gran desafío teórico pasó a ser la construcción de grandes sistemas ateóricos (como el DSM) que permitieran identificar los trastornos sobre los que se supone que cada uno de los remedios puede actuar más específicamente.

Aunque la perspectiva instaurada en los ochenta siga siendo hegemónica y permanezca incuestionada en los órganos de expresión de la comunidad psiquiátrica y, por supuesto, en las instituciones académicas, lo cierto es que hoy tenemos datos suficientes para sostener que ha resultado un fracaso. Los remedios que se suponía que iban a ser cada vez más específicos para trastornos cada vez más precisamente definidos, han resultado ser todo menos específicos. Recuérdese que los inhibidores selectivos de la recaptación de la serotonina (ISRS ) pretendían haberse convertido en la «bala de plata» que actuaba contra lo que se suponía que era la alteración específica de la depresión, frente a la inespecificidad de los antiguos -y tan baratos- antidepresivos tricíclicos. Incluso se crearon categorías diagnósticas nuevas -la depresión atípica- para excluir los trastornos sufridos por algunos pacientes que no respondían bien a 
EDITORIAL

los nuevos remedios. Hoy, los ISRS son el tratamiento farmacológico de primera elección de la depresión, pero también del trastorno de angustia, de la ansiedad generalizada, del trastorno obsesivo compulsivo, de los trastornos de la personalidad, de los trastornos del control de impulsos y de otros muchos. Si tenemos en cuenta, a la vez, que a los antipsicóticos responden los síntomas positivos de los pacientes esquizofrénicos, los delirios crónicos, los cuadros maníacos, los síntomas psicóticos de los trastornos mentales orgánicos y otros, quizás podíamos pensar que, aunque sólo fuera en consideración de lo que podemos aprender sobre nuestro trabajo como clínicos prescriptores -necesitaríamos articular nuestras clasificaciones- o, mucho mejor, pensar sobre la salud mental y los trastornos mentales sobre nuevas bases.

En los últimos años se han producido algunas señales de que existe una nueva preocupación social por la salud mental y sus alteraciones al menos en lo que solemos llamar el mundo desarrollado. Sin hacer mención a la proliferación de instrumentos de autoayuda que pretenden responder a la necesidad subjetivamente experimentada por multitudes de preservar su salud mental, si atendemos sólo a las manifestaciones institucionales encontramos que la salud y los trastornos mentales han vuelto a ser motivo de preocupación política, al menos en Europa.

La Organización Mundial de la Salud nos ha provisto, a través del proyecto ATLAS, de una visión de conjunto que nos permite conocer la situación a grandes rasgos de la atención a la salud mental en todo el mundo.
La Oficina Regional Europea de la Organización Mundial de la Salud propició el acuerdo entre veinticinco ministros de sanidad europeos, materializado en los llamados acuerdos de Helsinki, para tomar medidas concretas tendentes a mejorar la salud mental y actuar contra sus alteraciones en todos ellos. La Comisión Europea, a su vez, ha promovido la redacción del llamado Libro Verde, que reúne recomendaciones para articular una política de salud mental conjunta en Europa. El Consejo de Europa ha desarrollado importantes documentos y trabaja en el desarrollo de nuevos instrumentos que pretenden servir para proteger los derechos de las personas con trastornos mentales.

Algunos gobiernos, como el británico, han incrementado los fondos dedicados a la atención a la salud mental de un modo muy significativo, poniendo en marcha programas por los que han visto la luz, además de los importantes recursos ya existentes, los equipos de tratamiento asertivo comunitario, los equipos de atención en crisis o los equipos de atención temprana. El 31 de julio, el ministro de sanidad de ese país anunciaba el funcionamiento de los primeros equipos del plan por el que el Servicio Nacional de Salud va a dotarse de los psicoterapeutas necesarios para ofrecer psicoterapia como tratamiento de rutina para pacientes con ansiedad o depresión. Y la prestigiosa revista médica The Lancet, ha dedicado una serie de artículos haciéndose eco de todo lo anterior y proponiendo vías de actuación a través de una serie de artículos redactados por un llamado Lancet Global Mental 
EDITORIAL

Health Group, que reúne a 38 expertos internacionales en el tema.

A la vista de estos informes (alguno de los cuales utiliza datos relativamente antiguos), España se encuentra entre los países europeos que menos recursos dedica a la atención a la salud mental. La estructura del Estado de las Autonomías, con la circunstancia de que la mayor parte de las competencias sanitarias están transferidas, hace que no sea fácil trasponer las directivas europeas. Sin embargo, muy recientemente se han producido algunos avances importantes propiciados desde el gobierno central. En primer lugar se ha regulado por ley una cartera de servicios del sistema nacional de salud, que obliga a las autonomías y que define las responsabilidades en la materia de la atención primaria de salud y la atención especializada.

Dicha cartera incluye los tratamientos farmacológicos, la psicoterapia y la rehabilitación, lo que, hasta ese momento, era objeto de discusión.

Además, a iniciativa del Ministerio, el Consejo Interterritorial ha aprobado la Estrategia de Salud Mental del Sistema Nacional de Salud. Se trata de un documento que es, necesariamente, un documento de mínimos, porque expresa un acuerdo de todas las comunidades autónomas (un acuerdo, sorprendente en los tiempos que corren, y por ello, una buena noticia). Pero es un documento suficiente que, sin duda, servirá para armonizar el desarrollo de los servicios autonómicos y mejorar su calidad.

Una de las constantes en los documentos europeos es la constatación de que la salud mental, globalmente considerada, no puede ser una tarea sólo sanitaria sino que requiere de un trabajo coordinado en el que deben participar muchos otros departamentos. El documento titulado «Modelo de Atención en el Sistema para la Autonomía del Enfermo Mental Grave» publicado por el IMSERSO y fruto también de un amplio consenso, abre el camino a esta perspectiva, que debería extenderse necesariamente a otros departamentos, con especial urgencia a los responsables de las prisiones y la justicia, donde se dan situaciones inaceptables.

Este resurgir del tema no está exento de peligros. A los profesionales de la salud mental nos toca promover el debate social que permita sortear alguno de ellos: la delimitación del objeto de nuestro trabajo como clínicos (los trastornos mentales, ¿definidos con qué criterio?) del malestar general y de los comportamientos antiéticos, los obstáculos sociales, culturales y normativos a la integración y recuperación de las personas que sufren trastornos mentales, el uso coercitivo de los instrumentos de la psiquiatría (véase el nuevo protocolo de repatriación de emigrantes), los nuevos modos de vulneración de los derechos de los enfermos mentales. Con toda seguridad, oiremos hablar de ellos. 